\title{
COSMOGRAIL: the COSmological MOnitoring of GRAvltational Lenses
}

\section{Redshift of the lensing galaxy in seven gravitationally lensed quasars ${ }^{\star}$}

\author{
A. Eigenbrod, F. Courbin, and G. Meylan
}

\begin{abstract}
Laboratoire d'Astrophysique, École Polytechnique Fédérale de Lausanne (EPFL), Observatoire, 1290 Sauverny, Switzerland e-mail: alexander.eigenbrod@epfl.ch
\end{abstract}

Received 15 December 2006 / Accepted 12 January 2007

\section{ABSTRACT}

\begin{abstract}
Aims. The knowledge of the redshift of a lensing galaxy that produces multiple images of a background quasar is essential to any subsequent modeling, whether related to the determination of the Hubble constant $H_{0}$ or to the mass profile of the lensing galaxy. We present the results of our ongoing spectroscopic observations of gravitationally lensed quasars in order to measure the redshift of their lensing galaxies. We report on the determination of the lens redshift in seven gravitationally lensed systems.

Methods. Our deep VLT/FORS1 spectra are spatially deconvolved in order to separate the spectrum of the lensing galaxies from the glare of the much brighter quasar images. Our observing strategy involves observations in Multi-Object-Spectroscopy (MOS) mode which allows the simultaneous observation of the target and of several crucial PSF and flux calibration stars. The advantage of this method over traditional long-slit observations is that it allows a much more reliable extraction and flux calibration of the spectra. Results. We obtain the first reliable spectra of the lensing galaxies in six lensed quasars: FBQ 0951+2635 $\left(z_{\text {lens }}=0.260\right)$, BRI 0952-0115 ( $\left.z_{\text {lens }}=0.632\right)$, HE 2149-2745 ( $\left.z_{\text {lens }}=0.603\right)$, Q 0142-100 $\left(z_{\text {lens }}=0.491\right)$, SDSS J0246-0825 $\left(z_{\text {lens }}=0.723\right)$, and SDSS J0806+2006 $\left(z_{\text {lens }}=0.573\right)$. The last three redshifts also correspond to the Mg II doublet seen in absorption in the quasar spectra at the lens redshift. Our spectroscopic redshifts of HE 2149-2745 and FBQ 0951+2635 are higher than previously reported, which means that $H_{0}$ estimates from these two systems must be revised to higher values. Finally, we reanalyse the blue side of our previously published spectra of Q 1355-2257 and find $\mathrm{Mg}$ II in absorption at $z=0.702$, confirming our previous redshift estimate. The spectra of all lenses are typical of early-type galaxies.
\end{abstract}

Key words. gravitational lensing - cosmology: cosmological parameters

\section{Introduction}

About 100 gravitationally lensed quasars have been found since the discovery of the first case by Walsh et al. (1979). An advantage of lensed quasars resides in their possible variability, potentially leading to the measurement of the so-called time delay between the lensed images of the source. This quantity is directly related to the Hubble constant $H_{0}$ and to the slope of the mass profile of the lensing galaxy at the position of the quasar images projected on the plane of the sky. Assuming a model for the mass distribution in the lensing galaxy, and measuring the time delay, one can infer the value of $H_{0}$ (Refsdal 1964). Conversely, for a given $H_{0}$ estimate, the mass distribution in the lens can be reconstructed from the time delay measurement. In both cases, one wishes to construct a large, statistically significant sample of lensed quasars, either to reduce the random errors on $H_{0}$, or to build a large sample of massive early-type galaxies, the mass profile of which can be strongly constrained, thanks to gravitational lensing.

For whatever subsequent application of quasar lensing, some of the key measurements to carry out in each individual system are (i) the value of the time delay; (ii) the astrometry of all quasar images with respect to the lensing galaxy; and (iii) the redshift of the lensing galaxy.

^ Based on observations made with the ESO-VLT Unit Telescope 2 Kueyen (Cerro Paranal, Chile; Programs 077.A-0155, PI: G. Meylan).
The main goal of COSMOGRAIL (e.g. Eigenbrod et al. 2005 ) is to measure a large number of time delays from a photometric monitoring campaign based on a few 2-m class telescopes. While this goal can be reached only in the long run, it is possible to pave the way to accurate modeling of the systems by obtaining the redshift of the lensing galaxies in all the systems currently monitored. This is the goal of the present paper, which is the continuation of our spectroscopic study of lensed quasars, undertaken at the VLT with FORS1 (e.g. Eigenbrod et al. 2006b).

\section{VLT spectroscopy}

\subsection{Observations}

We present new observations of six gravitationally lensed quasars, in order to determine the redshift of the lensing galaxy, plus a re-analysis of one object previously published in Eigenbrod et al. (2006b). The details of the observational setup and of the data reduction can be found in Eigenbrod et al. (2006a,b), but we remind in the following some of the most important points.

Our observations are acquired with the FOcal Reducer and low dispersion Spectrograph (FORS1), mounted on the ESO Very Large Telescope at the Observatory of Paranal (Chile). All the observations are carried out in the MOS mode (Multi Object Spectroscopy). This strategy is the most convenient to get 
simultaneous observations of the main target and of several stars used both as flux calibrators and as reference point-spread functions (PSF) in order to spatially deconvolve the spectra. We choose these stars to be located as close as possible to the gravitationally lensed quasars with similar apparent magnitudes.

All targets are observed with the high-resolution collimator, allowing us to observe simultaneously eight objects over a field of view of $3.4^{\prime} \times 3.4^{\prime}$ with a pixel scale of $0.1^{\prime \prime}$. The GG435 order sorting filter in combination with the G300V grism is used for all objects, giving a useful wavelength range $4450<\lambda<$ $8650 \AA$ and a scale of $2.69 \AA$ per pixel in the spectral direction. This setup has a spectral resolution $R=\lambda / \Delta \lambda \simeq 200$ at the central wavelength $\lambda=5900 \AA$ for a $1.0^{\prime \prime}$ slit width in the case of the high resolution collimator. The choice of this grism favors spectral coverage rather than spectral resolution as we aim at measuring unknown lens redshifts.

We choose slitlets of $1.0^{\prime \prime}$ width, matching the seeing requested for these service-mode observations. Our observing sequences consist of a short acquisition image, an "image-throughslit" check, followed by two consecutive deep spectroscopic exposures. All individual exposures for all objects are $1400 \mathrm{~s}$ long. The journals of the observations are given in Table 1 . The through-slit images are displayed in Figs. 1 to 6, where the epochs refer to the exposure numbers in Table 1.

For every object we center at least two slitlets on foreground stars and one slitlet along the lensed images of the quasar. The mask is rotated to a Position Angle that avoids clipping of any quasar image. This is mandatory to carry out spatial deconvolution of the spectra. The spectra of the PSF stars are also used to cross-calibrate the flux scale as the data are taken at different airmasses and at different epochs (see Eigenbrod et al. 2006a). Six spectrophotometric standard stars are used to carry out the relative flux calibration, i.e. GD 108, HD 49798, LTT 377, LTT 1020, LTT 1788, and LTT 7987.

\subsection{Reduction and deconvolution}

We follow exactly the same procedure as described by Eigenbrod et al. (2006b). We carry out the standard bias subtraction, flat field correction and sky background subtraction using IRAF $^{1}$. The wavelength calibration is obtained from the spectrum of helium-argon lamps. The cosmic rays are removed using multiple exposures.

A flux cross-calibration of the spectra is applied before combining them into one final spectrum. This is done efficiently using the spectra of the PSF stars as references.

In order to separate the spectrum of the lensing galaxy from the spectra of the much brighter quasar images, we use the spectral version of the MCS deconvolution algorithm (Magain et al. 1998; Courbin et al. 2000)

This software uses the spatial information contained in the spectra of several reference PSF stars. The deconvolved spectra are sharpened in the spatial direction, and also decomposed into a "point-source channel" containing the spectra of the quasar images, and an "extended channel" containing the spectra of everything in the image which is not a point-source, i.e. in this case the spectrum of the lensing galaxy.

The deconvolved spectra of the lensing galaxies are extracted and smoothed with a 10 - $\AA$ box. Figures 7 to 12 display the

${ }^{1}$ IRAF is distributed by the National Optical Astronomy Observatories, which are operated by the Association of Universities for Research in Astronomy, Inc., under cooperative agreement with the National Science Foundation.
Table 1. Journal of the observations.

\begin{tabular}{|c|c|c|c|c|}
\hline$\overline{\mathrm{ID}}$ & $\overline{\text { Date }}$ & Seeing ["] & Airmass & Weather \\
\hline \multicolumn{5}{|c|}{ Q 0142-100 } \\
\hline 1 & $11 / 08 / 2006$ & 0.83 & 1.119 & Photometric \\
\hline 2 & $11 / 08 / 2006$ & 0.77 & 1.078 & Photometric \\
\hline 3 & $19 / 08 / 2006$ & 0.78 & 1.709 & Photometric \\
\hline 4 & $19 / 08 / 2006$ & 0.79 & 1.507 & Photometric \\
\hline \multicolumn{5}{|c|}{ SDSS J0246-0825 } \\
\hline 1 & $22 / 08 / 2006$ & 0.76 & 1.490 & Photometric \\
\hline 2 & $22 / 08 / 2006$ & 0.67 & 1.350 & Photometric \\
\hline 3 & $22 / 08 / 2006$ & 0.60 & 1.234 & Photometric \\
\hline 4 & $22 / 08 / 2006$ & 0.58 & 1.161 & Photometric \\
\hline 5 & $22 / 08 / 2006$ & 0.61 & 1.103 & Photometric \\
\hline 6 & $22 / 08 / 2006$ & 0.59 & 1.069 & Photometric \\
\hline \multicolumn{5}{|c|}{ SDSS J0806+2006 } \\
\hline 1 & $22 / 04 / 2006$ & 0.86 & 1.442 & Photometric \\
\hline 2 & $22 / 04 / 2006$ & 0.90 & 1.494 & Photometric \\
\hline 3 & $23 / 04 / 2006$ & 0.93 & 1.590 & Photometric \\
\hline 4 & $23 / 04 / 2006$ & 0.95 & 1.714 & Photometric \\
\hline \multicolumn{5}{|c|}{ FBQ $0951+2635$} \\
\hline 1 & $31 / 03 / 2006$ & 0.68 & 1.600 & Photometric \\
\hline 2 & $31 / 03 / 2006$ & 0.74 & 1.593 & Photometric \\
\hline 3 & $01 / 04 / 2006$ & 0.59 & 1.598 & Photometric \\
\hline 4 & $01 / 04 / 2006$ & 0.57 & 1.629 & Photometric \\
\hline \multicolumn{5}{|c|}{ BRI 0952-0115 } \\
\hline 1 & $23 / 04 / 2006$ & 0.67 & 1.097 & Photometric \\
\hline 2 & $24 / 04 / 2006$ & 0.58 & 1.087 & Photometric \\
\hline 3 & $24 / 04 / 2006$ & 0.56 & 1.094 & Photometric \\
\hline 4 & $24 / 04 / 2006$ & 0.50 & 1.116 & Photometric \\
\hline 5 & $24 / 04 / 2006$ & 0.42 & 1.165 & Photometric \\
\hline 6 & $24 / 04 / 2006$ & 0.45 & 1.225 & Photometric \\
\hline \multicolumn{5}{|c|}{ Q 1355-2257 } \\
\hline 1 & 05/03/2005 & 0.68 & 1.016 & Photometric \\
\hline 2 & 05/03/2005 & 0.73 & 1.040 & Photometric \\
\hline 3 & $20 / 03 / 2005$ & 0.63 & 1.038 & Photometric \\
\hline 4 & $20 / 03 / 2005$ & 0.54 & 1.015 & Photometric \\
\hline 5 & 20/03/2005 & 0.57 & 1.105 & Photometric \\
\hline 6 & $20 / 03 / 2005$ & 0.56 & 1.166 & Photometric \\
\hline \multicolumn{5}{|c|}{ HE 2149-2745 } \\
\hline 1 & 04/08/2006 & 0.66 & 2.004 & Photometric \\
\hline 2 & 04/08/2006 & 0.62 & 1.724 & Photometric \\
\hline 3 & 04/08/2006 & 0.62 & 1.461 & Photometric \\
\hline 4 & 04/08/2006 & 0.69 & 1.328 & Photometric \\
\hline 5 & $04 / 08 / 2006$ & 0.52 & 1.204 & Photometric \\
\hline 6 & $04 / 08 / 2006$ & 0.59 & 1.134 & Photometric \\
\hline
\end{tabular}

final one-dimensional spectra, where the Ca II H \& $\mathrm{K}$ absorption lines are obvious, as well as the 4000 - $\AA$ Balmer break, and the $\mathrm{G}$ band typical for $\mathrm{CH}$ absorption. In some cases, we identify a few more features that are labeled in the individual figures. The identified lines are used to determine the redshift of the lensing galaxies given in Table 2 . We compute the $1-\sigma$ error as the standard deviation between all the measurements of the individual lines. The absence of emission lines in all spectra indicates that these observed lensing galaxies are gas-poor early-type galaxies.

In most cases, no trace of the quasar broad emission lines is seen in the spectrum of the lensing galaxy, indicative of an accurate decomposition of the data into the extended (lens) and point source (quasar images) channels. Only our VLT spectrum of the lensing galaxy of BRI 0952-0115 is suffering from residuals of the quasar Ly $\alpha$ emission: the presence of the strong Ly $\alpha$ in the blue side of the spectrum complicates the deconvolution process. 

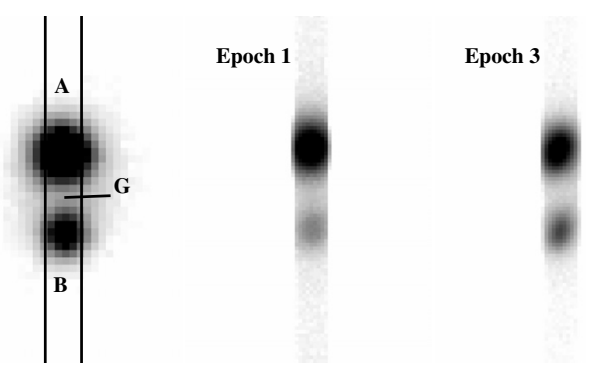

Fig. 1. Q 0142-100. Slit width: $1.0^{\prime \prime}$. Mask PA: $+75^{\circ}$.

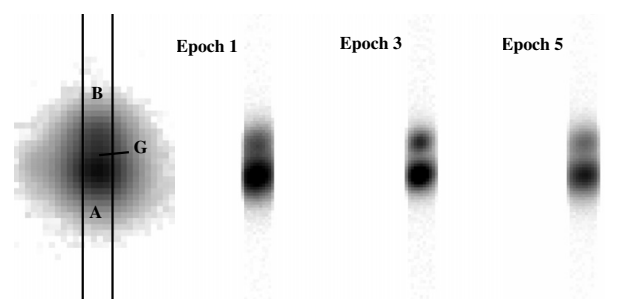

Fig. 2. SDSS J0246-0825. Slit width: $1.0^{\prime \prime}$. Mask PA: $+55^{\circ}$.

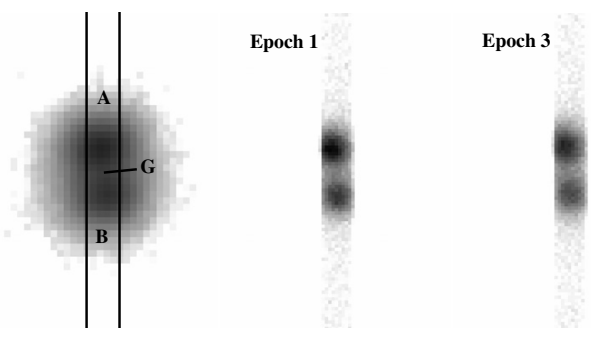

Fig. 3. SDSS J0806+2006. Slit width: $1.0^{\prime \prime}$. Mask PA: $-56^{\circ}$.

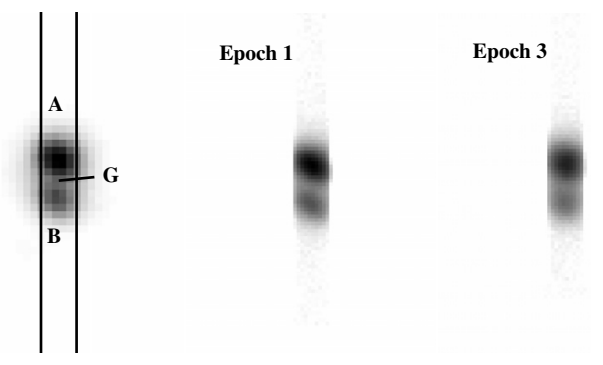

Fig. 4. FBQ 0951+2635. Slit width: $1.0^{\prime \prime}$. Mask PA: $+53^{\circ}$.

\section{Notes on individual objects}

Q 0142-100 (UM 673 or PHL 3703): this object was first discovered by Macalpine \& Feldman (1982) as a high redshift quasar with $z=2.719$. A few years later Surdej et al. (1987) identified Q 0142-100 as a gravitational lens with two quasar components separated by $2.2^{\prime \prime}$. No spectrum of the lensing galaxy has ever been obtained, but $\mathrm{Ca}$ II and $\mathrm{Na}$ I absorption lines are detected in the spectrum of the fainter quasar image B, suggesting that it is located at a redshift $z_{\text {lens }}=0.493$ (Surdej et al. 1988). From our spectrum of the lensing galaxy we confirm that it is the object responsible for the quasar absorption lines. Our best estimate of the redshift is $z_{\text {lens }}=0.491 \pm 0.001$. The observed galaxy spectrum is typical for an early type galaxy.

SDSS J0246-0825: this double-image quasar was discovered in the course of the Sloan Digital Sky Survey (SDSS) by Inada et al. (2005). The quasar has a redshift $z=1.68$ and an image separation of $1.04^{\prime \prime}$. From the Mg II and Mg I absorption lines observed in the spectra of the two quasar

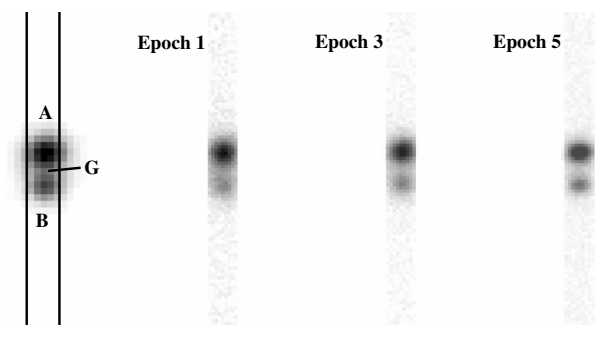

Fig. 5. BRI 0952-0115. Slit width: $1.0^{\prime \prime}$. Mask PA: $-45^{\circ}$.

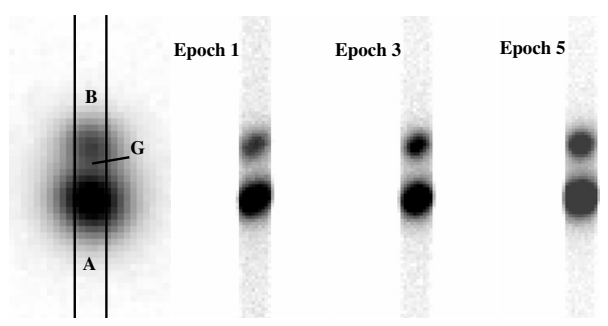

Fig. 6. HE 2149-2745. Slit width: $1.0^{\prime \prime}$. Mask PA: $-32^{\circ}$.

components, Inada et al. (2005) estimate the redshift of the lensing galaxy to be $z_{\text {lens }}=0.724$. Our direct redshift measurement $z_{\text {lens }}=0.723 \pm 0.002$ is in very good agreement with this value. The spectrum matches well that of an elliptical galaxy.

SDSS J0806+2006: this two-image gravitationally lensed quasar $(z=1.540)$ was recently discovered by Inada et al. (2006). The two quasar images are separated by $1.40^{\prime \prime}$. Several absorption lines are found in the quasar spectra, such as the (Mg II, Ca II H\&K and Fe II) lines, at a redshift of $z_{\text {lens }}=0.573$. The redshift we obtain from our spectrum is $z_{\text {lens }}=0.573 \pm 0.001$. The spectrum (see Fig. 9) matches very well the template spectrum of an elliptical galaxy.

FBQ 0951+2635: a double-image quasar at $z=1.246$ with $1.1^{\prime \prime}$ image separation found in the course of the FIRST Bright QSO Survey (FBQS) by Schechter et al. (1998). A time delay estimate of $\Delta t=16 \pm 2$ days between the quasar images is given by Jakobsson et al. (2005), but the redshift of the lensing galaxy, by the same authors, is found to be elusive. Schechter et al. (1998) detect absorption lines attributed to $\mathrm{Mg}$ II at $z=0.73$ and $z=0.89$ in both spectra of the quasar components. From the position of the lens on the fundamental plane, Kochanek et al. (2000) suggest $z_{\text {lens }} \simeq 0.21$. We measure $z_{\text {lens }}=0.260 \pm 0.002$ for this early-type lensing galaxy. This also corresponds to the photometric redshift of 0.27 measured by Williams et al. (2006) for a group of galaxies in the field of the lensing galaxy.

BRI 0952-0115: this gravitational lens was discovered by McMahon et al. (1992) as a pair of $z=4.50$ quasars separated by $0.9^{\prime \prime}$. Kochanek et al. (2000) found that the lens galaxy appears to be a typical early-type lens galaxy, with a fundamental plane redshift estimate of $z_{\text {lens }} \simeq 0.41$. We measure a significantly higher value, namely $z_{\text {lens }}=0.632 \pm 0.002$ (Fig. 11), and the spectrum of the lensing galaxy is well matched by that of an elliptical galaxy. With this new redshift, the lensing galaxy can no longer be considered as a member of the group of galaxies identified by Momcheva et al. (2006), but possibly of another small group in the field at $z \simeq 0.64$.

Q 1355-2257 (CTQ 327): this two-image quasar, discovered by Morgan et al. (2003), has a redshift of $z=1.373$, and an image separation of $1.23^{\prime \prime}$. The redshift of the lensing galaxies has been estimated by Morgan et al. (2003) to lie in the range $0.4<z_{\text {lens }}<0.6$. Ofek et al. (2005) note that their spectrum of the quasar component B shows an excess of emission longward 


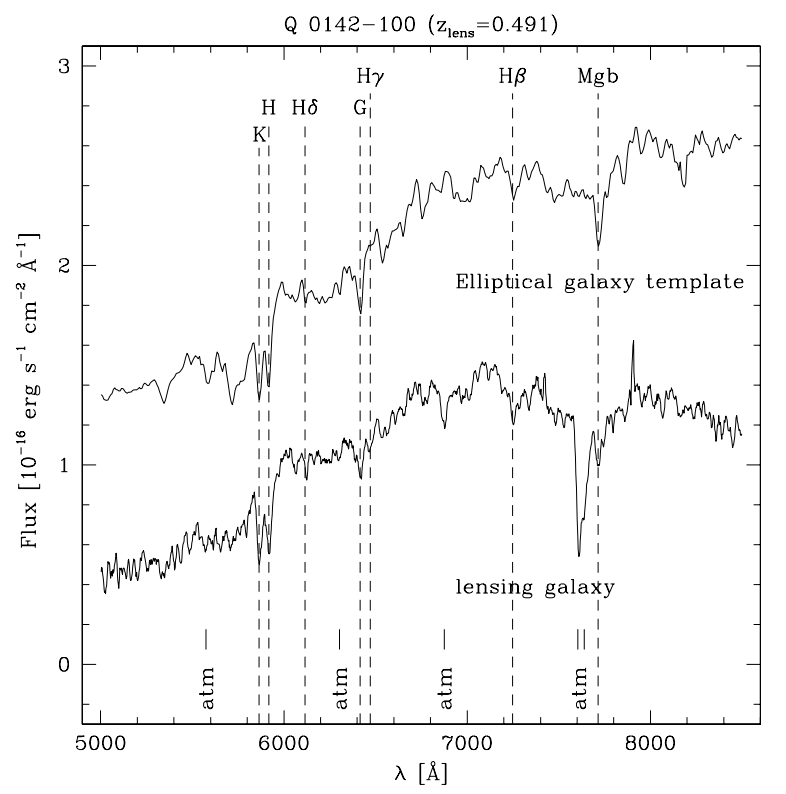

Fig. 7. Spectrum of the lens in Q 0142-100. The total integration time is $5600 \mathrm{~s}$. The template spectrum of a redshifted elliptical galaxy is shown for comparison (Kinney et al. 1996). Atmospheric absorptions are indicated in all figures by the label atm.

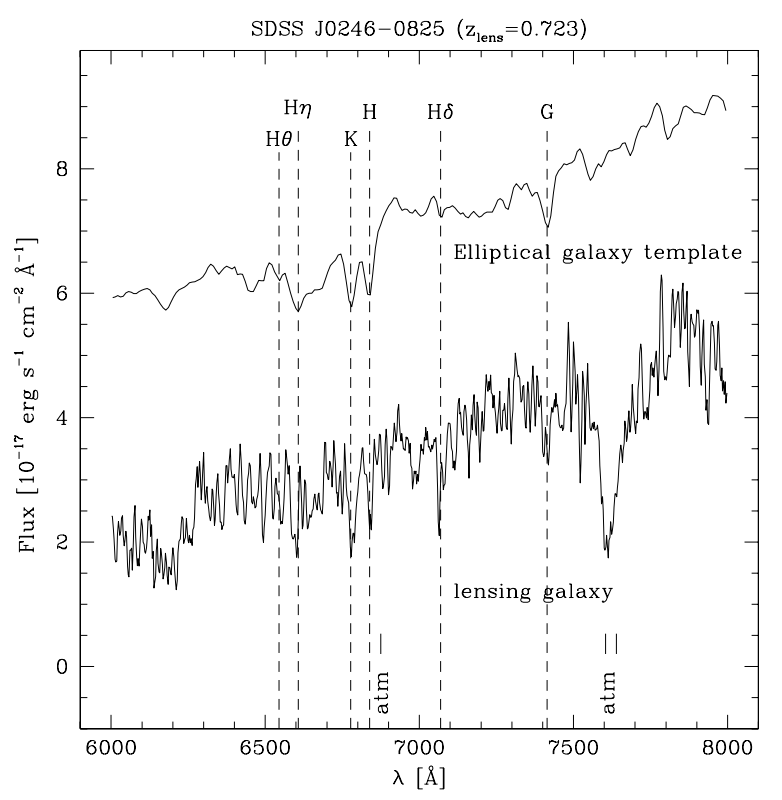

Fig. 8. Spectrum of the lens in SDSS J0246-0825. The total integration time is $8400 \mathrm{~s}$.

of $\sim 5870 \AA$. They associate the location of this emission excess to the 4000 - $\AA$ break of the lensing galaxy leading to a redshift of $z_{\text {lens }}=0.48$. In a previous paper (Eigenbrod et al. 2006b), we gave a significantly higher tentative redshift value of $z_{\text {lens }}=$ 0.701 . A re-analysis of our data allows to extend slightly our spectra to the blue side and to unveil the Mg II absorption line in the spectrum of the quasar component $\mathrm{B}$. The absorption is seen at the redshift we estimate for the spectrum of the lens, i.e. $z_{\text {lens }}=0.702 \pm 0.001$. Figure 13 displays the new quasar spectrum with an enlargement on the region around the $\mathrm{Mg}$ II absorption.

HE 2149-2745: Wisotzki et al. (1996) found this bright gravitationally lensed quasar at $z=2.033$, with two images separated by $1.71^{\prime \prime}$. They gave first estimates of the redshift of the lensing galaxy, refined later by Lopez et al. (1998), who infer

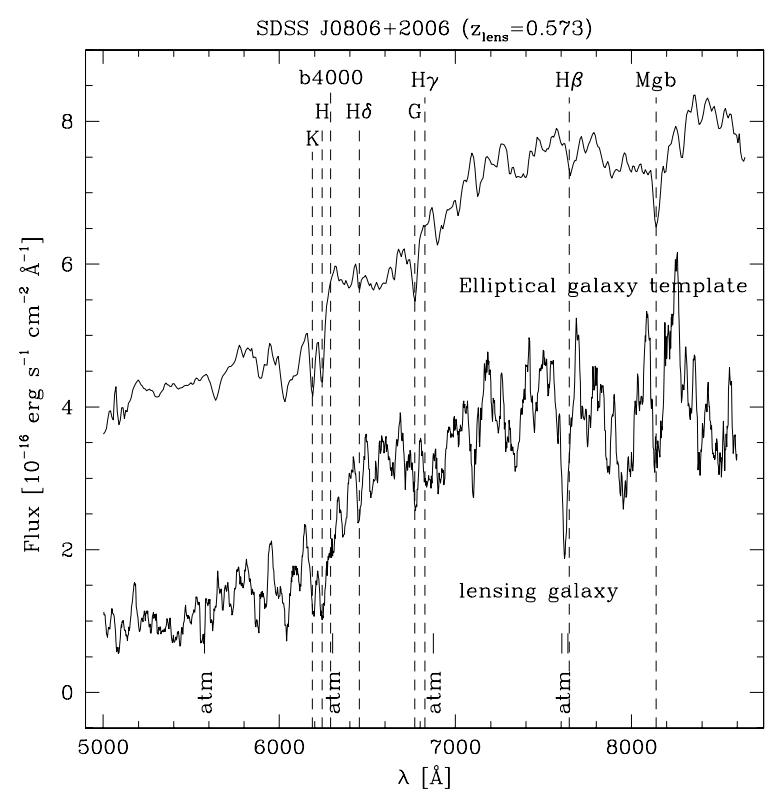

Fig. 9. Spectrum of the lens in SDSS J0806+2006. The total integration time is $5600 \mathrm{~s}$.

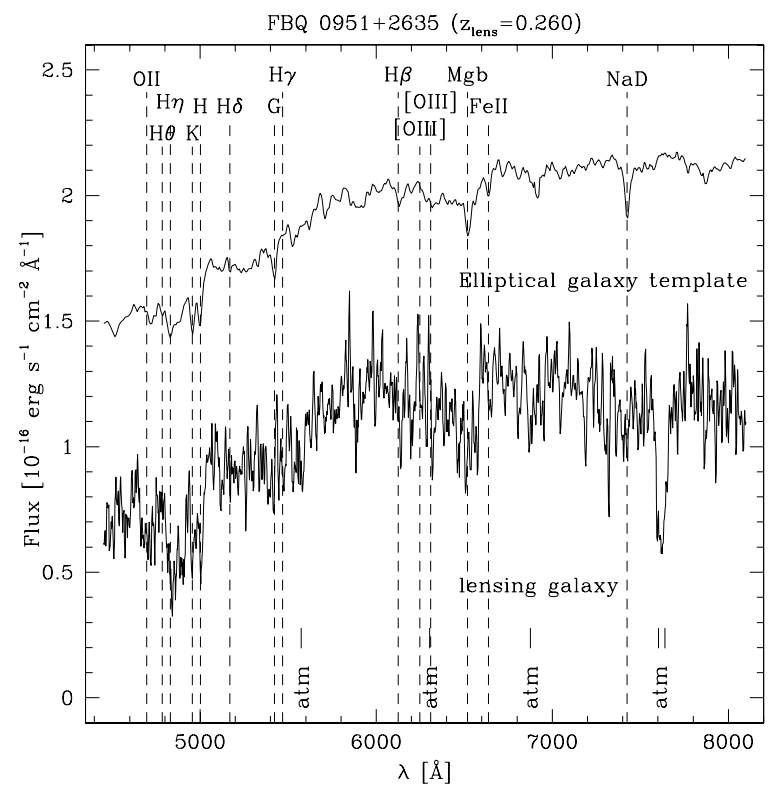

Fig. 10. Spectrum of the lens in FBQ 0951+2635. The total integration time is $5600 \mathrm{~s}$.

a probable redshift range of $\sim 0.3-0.5$. Kochanek et al. (2000) give a fundamental plane redshift estimate of $0.37 \leq z_{\text {lens }} \leq 0.50$. Burud et al. (2002) measure the time delay between the two images $(\Delta t=103 \pm 12$ days $)$ and report a tentative redshift of $z_{\text {lens }}=0.495 \pm 0.010$ by cross-correlating the lens spectrum with a template spectrum of an elliptical galaxy, but they notice that the signal-to-noise of the correlation function is poor. Our direct spectroscopic result disagrees with all previous estimates, with the new value of $z_{\text {lens }}=0.603 \pm 0.001$. This value corresponds to the photometric redshift $z \simeq 0.59$ (later spectroscopically confirmed at $z=0.603$ ) of a galaxy group in the field (Williams et al. 2006; Momcheva et al. 2006). Faure et al. (2004) also reported this group of galaxies at the photometric redshift $z=0.7 \pm 0.1$. 


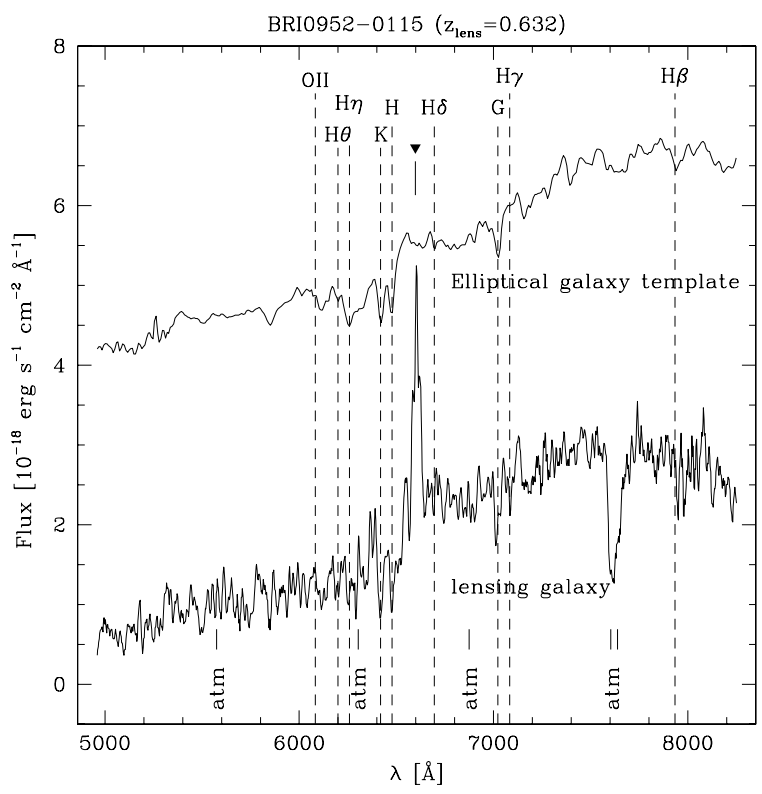

Fig. 11. Spectrum of the lens in BRI 0952-0115. The total integration time is $8400 \mathrm{~s}$. The emission feature marked by the black triangle is residual light of the quasar images. The Ly $\alpha$ emission of the quasar falls exactly at this wavelength.

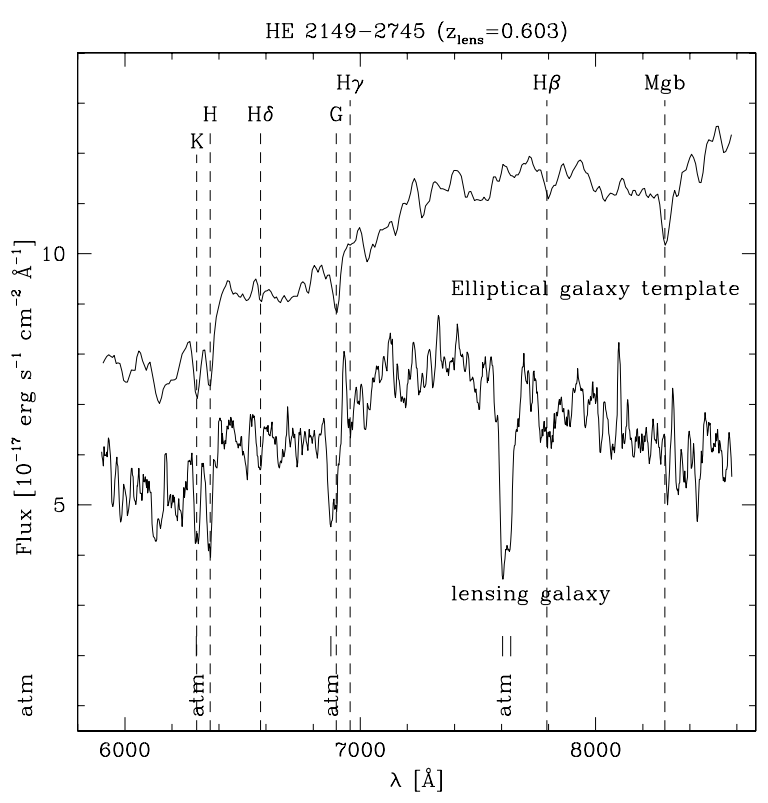

Fig. 12. Spectrum of the lens in HE 2149-2745. The total integration time is $8400 \mathrm{~s}$.

\section{Summary and conclusions}

We present straightforward VLT spectroscopic observations of six gravitationally lensed quasars and we measure the redshift of the lensing galaxy directly from the continuum light spectrum and several sets of absorption lines. The MOS mode in which all observations are taken and the subsequent observation of several PSF stars are crucial to carry out a reliable decontamination of the lens spectrum by those of the quasar images. The PSF stars are also used to carry out a very accurate flux calibration of the spectra.

Three of our redshifts measurements also correspond to absorption lines seen in the quasar spectrum. Following the work of Williams et al. (2006) and Momcheva et al. (2006), we
Table 2. Redshift values determined for the lensing galaxies in the seven gravitational lenses.

\begin{tabular}{lc}
\hline \hline Object & $z_{\text {lens }}$ \\
\hline Q 0142-100 & $0.491 \pm 0.001$ \\
SDSS J0246-0825 & $0.723 \pm 0.002$ \\
SDSS J0806+2006 & $0.573 \pm 0.001$ \\
FBQ 0951+2635 & $0.260 \pm 0.002$ \\
BRI 0952-0115 & $0.632 \pm 0.002$ \\
Q 1355-2257 & $0.702 \pm 0.001$ \\
HE 2149-2745 & $0.603 \pm 0.001$ \\
\hline
\end{tabular}

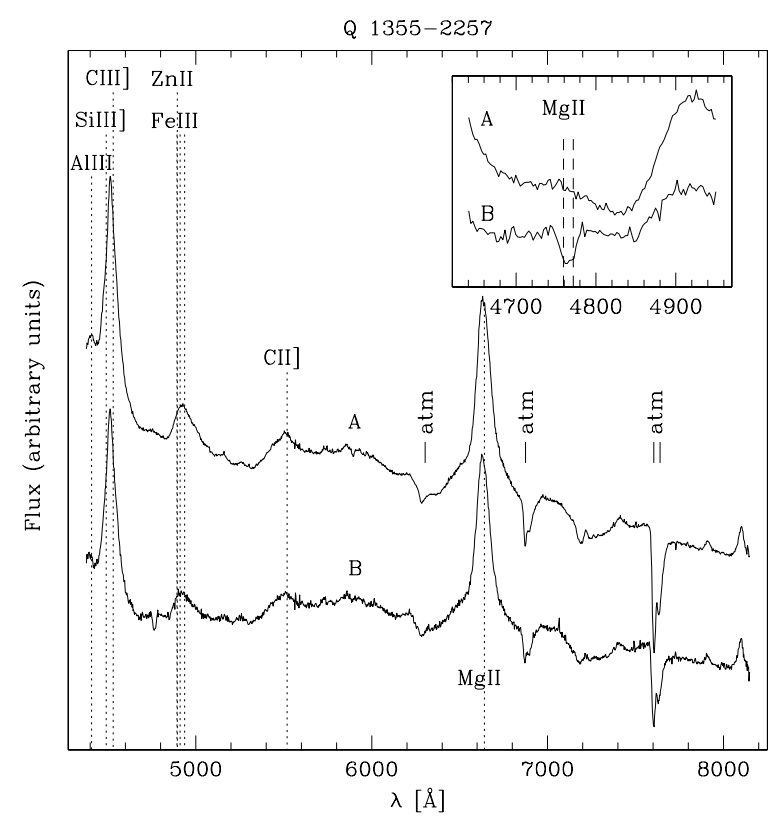

Fig. 13. Spectrum of the images of quasar Q 1355-2257. The total integration time is $8400 \mathrm{~s}$. In the inlet, we show the $\mathrm{Mg}$ II absorption lines present in the spectrum of component B and which is due to the lensing galaxy.

identify three lensing galaxies as members of small groups of galaxies. A re-analysis of the spectra of Q 1355-2257, which lens spectrum has a low signal-to-noise ratio in Eigenbrod et al. (2006b), allows to detect the Mg II doublet in absorption in the quasar spectrum and confirm our previous redshift estimate, i.e. $z_{\text {lens }}=0.702 \pm 0.001$.

Finally, our probably most exciting results are our new redshift measurements for the two lensed quasars with time-delay determinations FBQ $0951+2635$ and HE 2149-2745, that are not compatible with previous estimates. We find $z_{\text {lens }}=0.260 \pm$ 0.002 instead of $z_{\text {lens }}=0.21$ for the first and $z_{\text {lens }}=0.603 \pm 0.001$ for the second, instead of $z_{\text {lens }}=0.495 \pm 0.010$. This implies that previous estimates of $H_{0}$ based on these two systems must be revised to higher values, for a given lens model. While the impact of the change in redshift is negligible for FBQ 0951+2635 given the present uncertainties on the measured time delay (Jakobsson et al. 2005), it is sufficiently large in the case of HE 2149-2745 (Burud et al. 2002) to justify some new modeling of the system.

These new lens redshifts have a direct impact on several previous studies. More specifically the inferred value for $H_{0}$ from the multiple-lens models of Saha et al. (2006) should be updated with these new redshifts, as well as with the recently measured time delay of SDSS J1650+4251 by Vuissoz et al. (2007). In other studies (e.g. Kochanek 2002, 2003; Oguri 2006), HE 2149-2745 is one of lens systems that has been used to 
argue possible low $H_{0}$ values from time delays. The new lens redshift significantly increases the derived $H_{0}$, thereby weakening the possible low $H_{0}$ problem.

Acknowledgements. The authors are very grateful to the ESO staff at Paranal for the particular care paid to the slit alignment necessary to perform the spectra deconvolutions. COSMOGRAIL is financially supported by the Swiss National Science Foundation (SNSF).

\section{References}

Burud, I., Courbin, F., Magain, P., et al. 2002, A\&A, 383, 71 Courbin, F., Magain, P., Kirkove, M., \& Sohy, S. 2000, ApJ, 529, 1136

Eigenbrod, A., Courbin, F., Vuissoz, C., et al. 2005, A\&A, 436, 25

Eigenbrod, A., Courbin, F., Dye, S., et al. 2006a, A\&A, 451, 747

Eigenbrod, A., Courbin, F., Meylan, G., et al. 2006b, A\&A, 451, 759

Faber, S. M., \& Jackson, R. E. 1976, ApJ, 204, 668

Faure, C., Alloin, D., Kneib, J. P., et al. 2004, A\&A, 428, 741

Inada, N., Burles, S., Gregg, M. D., et al. 2005, AJ, 130, 1967

Inada, N., Oguri, M., Becker, R. H., et al. 2006, AJ, 131, 1934

Jakobsson, P., Hjorth, J., Burud, I., et al. 2005, A\&A, 431, 103
Kinney, A. L., Calzetti, D., Bohlin, R. C., et al. 1996, ApJ, 467, 38 Kochanek, C. S. 2002, ApJ, 578, 25

Kochanek, C. S. 2003, ApJ, 583, 49

Kochanek, C. S., Falco, E. E., Impey, C. D., et al. 2000, ApJ, 543, 131

Lopez, S., Wucknitz, O., \& Wisotzki, L. 1998, A\&A, 339, L13

Macalpine, G. M., \& Feldman, F. R. 1982, ApJ, 261, 412

Magain, P., Courbin, F., \& Sohy, S. 1998, ApJ, 494, 452

McMahon, R., Irwin, M., \& Hazard, C. 1992, Gemini, 36, 1

Momcheva, I., Williams, K. A., Keeton, C. R., et al. 2006, ApJ, 641, 169

Morgan, N. D., Gregg, M. D., Wisotzki, L., et al. 2003, AJ, 126, 696

Morgan, N. D., Cardwell, J. A. R., Schechter, P. L., et al. 2004, AJ, 127, 2617

Ofek, E. O., Maoz, D., Rix, H.-W., et al. 2006, ApJ, 641, 70

Oguri, M. 2006 [arXiv:astro-ph/0609694]

Refsdal, S. 1964, MNRAS, 128, 307

Saha, P., Coles, J., Maccio', A. V., et al. 2006, ApJ, 650, L17

Schechter, P. L., Gregg, M. D., Becker, R. H., et al. 1998, AJ, 115, 1371

Surdej, J., Swings, J.-P., Magain, P., et al. 1987, Nature, 329, 695

Surdej, J., Magain, P., Swings, J.-P., et al. 1988, A\&A, 198, 49

Vuissoz, C., Courbin, F., Sluse, D., et al. 2007, A\&A, 464, 845

Walsh, D., Carswell, R. F., \& Weymann, R. J. 1979, Nature, 279, 381

Williams, K. A., Momcheva, I., Keeton, C. R., et al. 2006, ApJ, 646, 85

Wisotzki, L., Koehler, T., Lopez, S., et al. 1996, A\&A, 315, 405 